\title{
L-Cysteine Adsorption Structures on Au(111) Investigated by Scanning Tunneling Microscopy under Ultrahigh Vacuum Conditions
}

\author{
Angelika Kühnle, ${ }^{\dagger}$ Trolle R. Linderoth,* Michael Schunack, and Flemming Besenbacher \\ Interdisciplinary Nanoscience Center (iNANO) and Department of Physics and Astronomy, \\ University of Aarhus, DK-8000 Aarhus C, Denmark
}

Received September 21, 2005. In Final Form: November 28, 2005

\begin{abstract}
Adsorption structures formed upon vapor deposition of the natural amino acid L-cysteine onto the (111) surface of gold have been investigated by scanning tunneling microscopy under ultrahigh vacuum conditions. Following deposition at room temperature and at cysteine coverages well below saturation of the first monolayer, we found coexistence of unordered molecular islands and extended domains of a highly ordered molecular overlayer of quadratic symmetry. As the coverage was increased, a number of other structures with local hexagonal order emerged and became dominant. Neither of the room temperature, as-deposited, ordered structures showed any fixed rotational relationship to the underlying gold substrate, suggesting a comparatively weak and nonspecific molecule-substrate interaction. Annealing of the cysteine-covered substrate to $380 \mathrm{~K}$ lead to marked changes in the observed adsorption structures. At low coverages, the unordered islands developed internal order and their presence started to perturb the appearance of the surrounding $\mathrm{Au}(111)$ herringbone reconstruction. At coverages beyond saturation of the first monolayer, annealing led to development of a $(\sqrt{ } 3 \times \sqrt{ } 3) R 30^{\circ}$ superstructure accompanied by the formation of characteristic monatomically deep etch pits, i.e., the behavior typically observed for alkanethiol self-assembled monolayers on $\mathrm{Au}(111)$. The data thus show that as-deposited and thermally annealed cysteine adsorption structures are quite different and suggest that thermal activation is required before vacuum deposited cysteine becomes covalently bound to single crystalline $\mathrm{Au}(111)$.
\end{abstract}

\section{Introduction}

Adsorption of biomolecules ${ }^{1}$ and chemical functionalization of surfaces by self-assembled monolayers (SAMs) ${ }^{2-4}$ are areas of investigation receiving much current attention. The interaction of the amino acid cysteine $\left(\mathrm{HS}-\mathrm{CH}_{2}-\mathrm{CH}\left(\mathrm{NH}_{2}\right)-\mathrm{COOH}\right)$ with gold surfaces is interesting from both of these perspectives: Thiols on $\mathrm{Au}(111)$ are the prototypical SAM system in which $\mathrm{S}-\mathrm{Au}$ covalent bonding anchors the molecules to the surface, ${ }^{2-4}$ and proteins ${ }^{5}$ or artificial peptides ${ }^{6}$ have been shown to bind to gold surfaces through sulfur atoms on cysteine residues.

Previous experimental investigations of cysteine adsorption on gold surfaces ${ }^{7-14}$ span a range of conditions from growth of monolayers in aqueous solution, sometimes containing $\mathrm{pH}_{-}$

* To whom correspondence should be addressed. Phone: +4589425536 Fax: +45 8942 3690. E-mail: trolle@inano.dk.

$\dagger$ Present address: Fachbereich Physik, Universität Osnabrück, Barbarastr. 7, D-49076 Osnabrück, Germany.

(1) Kasemo, B. Surf. Sci. 2002, 500, 656.

(2) Schreiber, F. Prog. Surf. Sci. 2000, 65, 151.

(3) Ulman, A. Chem. Rev. 1996, 96, 1533.

(4) Poirier, G. E.; Pylant, E. D. Science 1996, 272, 1145

(5) Chi, Q.; Zhang, J.; Nielsen, J. U.; Friis, E. P.; Chorkendorff, I.; Canters, G. W.; Andersen, J. E. T.; Ulstrup, J. J. Am. Chem Soc. 2000, 122, 4047.

(6) Baas, T.; Gamble, L.; Hauch, K. D.; Castner, D. G.; Sasaki, T. Langmuir 2002, 18,4898 .

(7) Uvdal, K.; Bodö, P.; Liedberg, B. J. Colloid Interface Sci. 1992, 149, 162. (8) Dakkouri, A. S.; Kolb, D. M.; Edelstein-Shima, R.; Mandler, D. Langmuir 1996, 12,2849

(9) Dodero, G.; Michieli, L. D.; Cavalleri, O.; Rolandi, R.; Oliveri, L.; Dacca, A.; Parodi, R. Colloids Surf. A 2000, 175, 121. 10.

(10) Yang, W.; Gooding, J. J.; Hibbert, D. B. J. Electroanal. Chem. 2001, 516,

(11) Cavalleri, O.; Oliveri, L.; Dacca, A.; Parodi, R.; Rolandi, R. Appl. Surf. Sci. 2001, 175-176, 357.

(12) Qingwen, L.; Hong, G.; Yiming, W.; Guoan, L.; Jie, M. Electroanalysis 2001, 13, 1342 .

(13) Zhang, J.; Chi, Q.; Nielsen, J. U.; Friis, E. P.; Andersen, J. E. T.; Ulstrup, J. Langmuir 2000, 16, 7229.

(14) Zhang, J.; Chi, Q.; Andersen, J. E. T.; Hansen, A. G.; Nielsen, J. U.; Ulstrup, J. Probe Microsc. 2001, 2, 151. controlling buffer salts and with or without electrochemical control, to vapor deposition in ultrahigh vacuum (UHV). Substrates range from poly-crystalline gold films to single-crystal surfaces. The primary characterization techniques have been in situ electrochemical scanning tunneling microscopy (STM) and X-ray photoelectron spectroscopy (XPS) but also thermal desorption spectroscopy (TDS) and various scattering techniques have been applied. Key issues addressed in these studies have been the nature of the cysteine-gold interaction, the chemical state of the adsorbed cysteine, and the structure of the cysteine overlayers.

Two separate XPS investigations have been performed on vapor $^{7}$ and solution ${ }^{9,11}$ deposited cysteine films. Both observed a shift of the $S 2 p_{3 / 2}$ peak attributed to a thiolate species covalently bound to gold, consistent with the general picture for alkanethiols on gold. ${ }^{2}$ A second component of the $S$ peak was assigned to unbound - -SH groups, suggesting that cysteine forms a double layer where the molecules in the second layer are oriented with their sulfur atoms away from the surface. The optimum sulfur adsorption site has been found to be the Au bridge site from theoretical modeling based on density functional theory (DFT). ${ }^{15}$ From these calculations, it was furthermore found that the binding of cysteine becomes stronger if also the $\mathrm{N}$ atom of the amino group interacts with the surface. A similar conclusion was drawn from early infrared spectroscopy measurements ${ }^{16}$ but was not observed in later XPS studies., ${ }^{9,11}$ Thermal desorption spectroscopy on vapor deposited films ${ }^{17}$ has shown intact cysteine molecules to desorb from multilayers at $353-373 \mathrm{~K}$, whereas fragment peaks at 458 and $478 \mathrm{~K}$ were assigned to decomposition products from the first (covalently bound) monolayer. This study also

(15) Felice, R. D · Selloni, A ; Molinari, E. J. Chem. Phys. B 2003, 107, 1151

(16) Ihs, A.; Liedberg, B. J. Colloid Interface Sci. 1991, 144, 282.

(17) Shin, T.; Kim, K.-N.; Lee, C.-W.; Shin, S. K.; Kang H. J. Phys. Chem. 2003, 107, 11674 
concluded that the cysteine atoms in the first monolayer are in zwitterionic form, consistent with earlier XPS studies. ${ }^{9}$

The lateral order in cysteine/Au(111) adlayers has been addressed by STM with somewhat deviating results. $8,9,13,18$ Two studies in air ${ }^{9}$ and liquid ${ }^{8}$ found structures with $(\sqrt{ } 3 \times \sqrt{ } 3)$ R3 $30^{\circ}$ order consistent with the behavior of long- ${ }^{2}$ and short-chained ${ }^{19}$ alkanethiols. (Also the characteristic monatomically deep etchpits found upon thiol SAM formation was observed. ${ }^{9}$ ) In contrast, Zhang et al. found by electrochemical methods that the coverage in saturated monolayers was inconsistent with a $(\sqrt{ } 3 \times \sqrt{3})$ $\mathrm{R} 30^{\circ}$ structure and their high-resolution STM data were interpreted as a hydrogen-bonded network with 6 molecules in a $(3 \sqrt{ } 3 \times 6) \mathrm{R} 30^{\circ}$ supercell.

The scope of the present paper is to present the first investigation by STM of adsorption structures formed from cysteine on Au(111) under ultrahigh vacuum conditions. It is important to extend the database on adsorption structures to such ultra-clean conditions where a solvent medium, which may affect the intermolecular interactions and hence the ordering on the surface, is absent. Vacuum deposition furthermore offers improved control of the molecular coverage, from the submonolayer to multilayer range, and the substrate temperature becomes a parameter which can be varied.

\section{Experimental Methods}

The adsorption experiments were performed in a UHV chamber equipped with the home-built Aarhus $\mathrm{STM}^{20}$ as well as standard facilities for sample cleaning and characterization. The surface of the $\mathrm{Au}(111)$ single crystal was cleaned by repeated cycles of $\mathrm{Ar}^{+}$ ion sputtering at $1.5 \mathrm{keV}$ and annealing at $800 \mathrm{~K}$ until a well-ordered $(22 \times \sqrt{ } 3)$ herringbone reconstruction ${ }^{21}$ was observed. The cysteine molecules were evaporated inside the vacuum chamber onto the clean gold substrate from a home-built evaporation source consisting of a small (approximately $3 \mathrm{~mm}$ ) quartz tube wound with a metal wire for resistive heating and with a $\mathrm{Cr} / \mathrm{Al}$ thermocouple pair fused into the quartz for temperature monitoring. During evaporation, the crucible was held at specific temperatures in the range $363-390 \mathrm{~K}$, resulting in cysteine coverages ranging from submonolayer to beyond saturation of the first layer using evaporation times of a few minutes (source-sample distance approximately $10 \mathrm{~cm}$ ). The cysteine (Aldrich, $97 \%$ L-cysteine) was used as received from the supplier but was thoroughly outgassed in the evaporator before deposition to remove volatile contaminants. In all of the experiments, the temperature of the $\mathrm{Au}(111)$ substrate during deposition was approximately $300 \mathrm{~K}$. To thermally anneal the deposited films, the temperature of the Au crystal was brought to a specific temperature in the range 353-398 $\mathrm{K}$ for 5 or $10 \mathrm{~min}$ by radiative heating of the backside of the sample holder using a W (halogen light-bulb) filament. All STM measurements were performed at room temperature (approximately $300 \mathrm{~K}$ ) in constant current mode $\left(I_{\mathrm{t}} \approx 0.1 \mathrm{nA}, V_{\mathrm{t}} \approx 1.5 \mathrm{~V}\right.$, sample at negative potential compared to tip).

\section{Results}

When cysteine is deposited on the $\mathrm{Au}(111)$ surface at room temperature, and at submonolayer coverages, nucleation is found to occur at the elbows of the herringbone reconstruction, leading to ordered arrays of molecular clusters as displayed in Figure 1. Elongated molecular islands grow from these nucleation sites, and from step edges, and with increasing coverage eventually cover large portions of the Au terraces (see inset in Figure 1).

(18) Xu, Q.-M.; et al. Langmuir 2001, 17, 6203.

(19) Paradis E.; Rowntree P. J. Electroanal. Chem. 2003, 550, 175

(20) Besenbacher, F. Rep. Prog. Phys. 1996, 59, 1737. See also www.specs.de

(21) Barth, J. V.; Brune, H.; Ertl, G.; Behm, R. J. Phys. Rev. B 1990, 42, 9307.

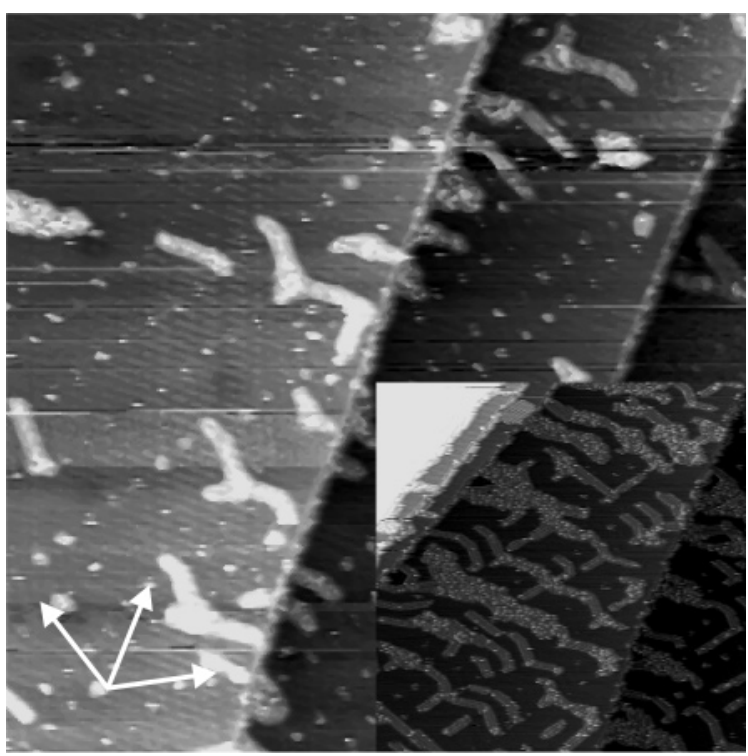

Figure 1. STM images acquired after evaporation of cysteine at room temperature. a: Nucleation in the elbows of the herringbone reconstruction and formation of unordered cysteine islands. The arrows mark the close-packed row directions of the gold surface. Image size: $1699 \AA \times 1740 \AA$. Inset: Upon increased exposure time the cysteine islands grow in size. Image size: $1110 \AA \times 1160$ A.

These islands display little or no internal order and their perimeters preferentially follow directions defined by the ridges of the herringbone reconstruction which is not perturbed by the presence of the islands.

Extended domains of a highly ordered structure are found to coexist with these unordered islands, often covering entire terraces. An STM image of this structure is shown in Figure 2a. The characteristic corrugation pattern of the herringbone reconstruction is clearly seen to modulate the height of this molecular overlayer, showing that the surface reconstruction is maintained in the gold substrate underneath. The overlayer has been observed in different imaging modes, ascribed to changes in the tip condition, as shown in the high-resolution images in panels $b$ and $c$ of Figure 2. Neglecting the height modulation caused by the underlying surface reconstruction, the overlayer has 4-fold rotational symmetry with a quadratic unit cell of $12 \AA$ side length. The unit cells marked in $\mathrm{b}$ and c cover similar areas and have been aligned based on images where the tip condition changes within one image. The unit cell in panel $b$ contains four bright features with a top-to-top distance of about $4 \AA$. We suggest that each of these protrusions corresponds to one molecule, rather than part of a molecule imaged with submolecular resolution, since an individual cysteine molecule is expected to have a footprint of approximately $5 \AA$ lateral extent, depending upon its orientation on the surface. The rotational orientation of the molecular overlayer with respect to the underlying substrate has been analyzed by determining the angle for a characteristic direction in the overlayer and comparing this to the angle for the close-packed [1-10] directions of the substrate as determined from atomically resolved images. As the underlying substrate exhibits 6-fold rotational symmetry, the measured angles are transferred to an interval from $0^{\circ}$ to $30^{\circ}$. This analysis shows that there is no preferential rotational relationship between substrate and overlayer. Even for domains observed in the same STM image (where possible distortions due to thermal drift can be ruled out), we find that the domain orientations can vary by up to $10^{\circ}$. This absence of epitaxial relationship points toward either 

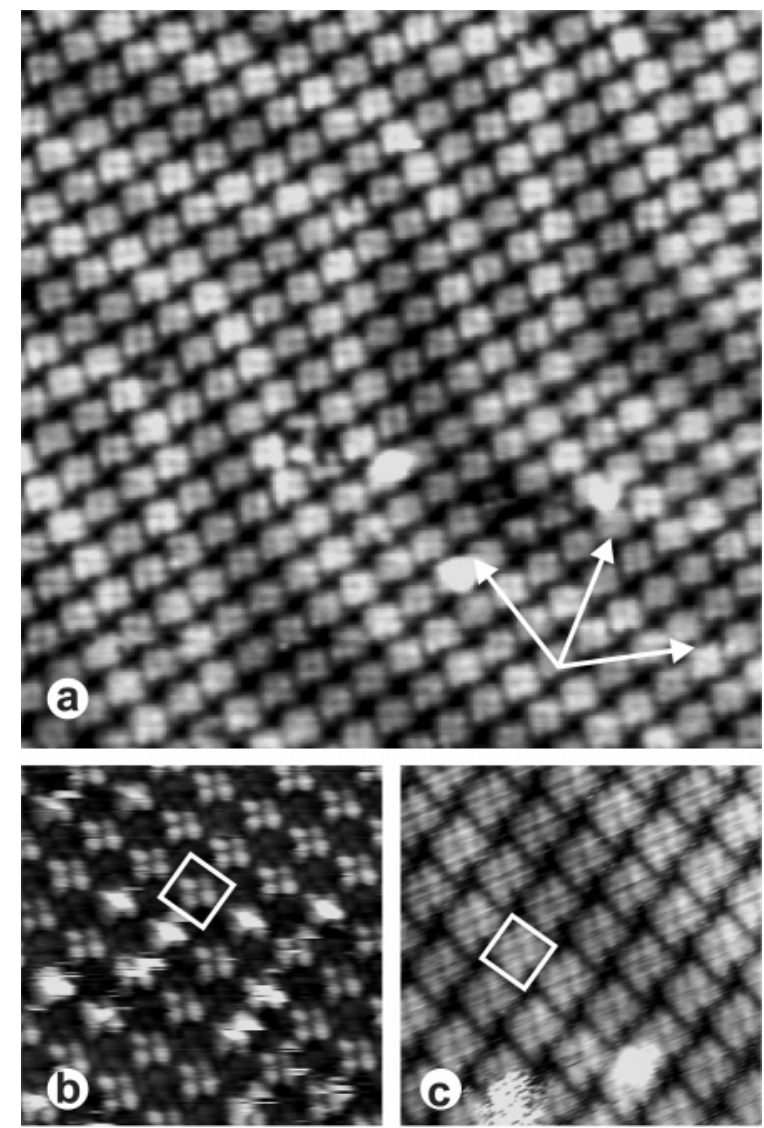

Figure 2. Ordered cysteine overlayer of quadratic symmetry formed upon room-temperature deposition. (a) Demonstration that the herringbone reconstruction of the $\mathrm{Au}(111)$ substrate is unperturbed by the molecular overlayer. Image size: $222 \AA \times 232 \AA$. (b and c) High-resolution images of the overlayer in two different imaging modes $(78 \AA \times 81 \AA)$. A quadratic unit cell with sidelength $d=$ $12 \AA$ is indicated.

a situation with a number of competing adsorption sites ${ }^{22}$ or a noncovalent molecule-surface interaction. Since the herringbone reconstruction is known to be easily perturbed upon chemisorption of adsorbates, and from the STM images appears to be unaffected by the cysteine overlayer, the latter possibility seems more likely.

In Figure 3, yet a third adsorption structure is shown. This phase coexists with the disordered islands and the 4-fold symmetric overlayer but becomes more pronounced at higher coverages. In this structure, the protrusions assume nearhexagonal order and their separation of $5.7 \pm 0.3 \AA$ again suggests they correspond to individual cysteine molecules. These dimensions of the hexagonal layer are close to those expected for a 2 $\times 2$ superstructure, but also for this structure, no fixed rotational relationship to the underlying substrate appears to exist. In certain areas, several cysteine molecules appear to be missing from the hexagonally packed overlayer, leading to dark rows with a characteristic zigzag appearance in the STM images (Figure 3b). The faint structures observed between the uppermost protrusions in this image indicate the structures in fact consist of double or even multiple layers of molecules.

Thermal annealing at $380 \mathrm{~K}$ of the sample covered by submonolayer quantities of cysteine leads to pronounced changes to the previously disordered, elongated islands (Figure 1), which now develop local order as demonstrated in Figure 4. The inset shows a zoom-in on an island displaying a characteristic structure in which parallelograms consisting of four bright features align

(22) Felice, R. D.; Selloni, A. J. Chem. Phys. 2004, 120, 4906
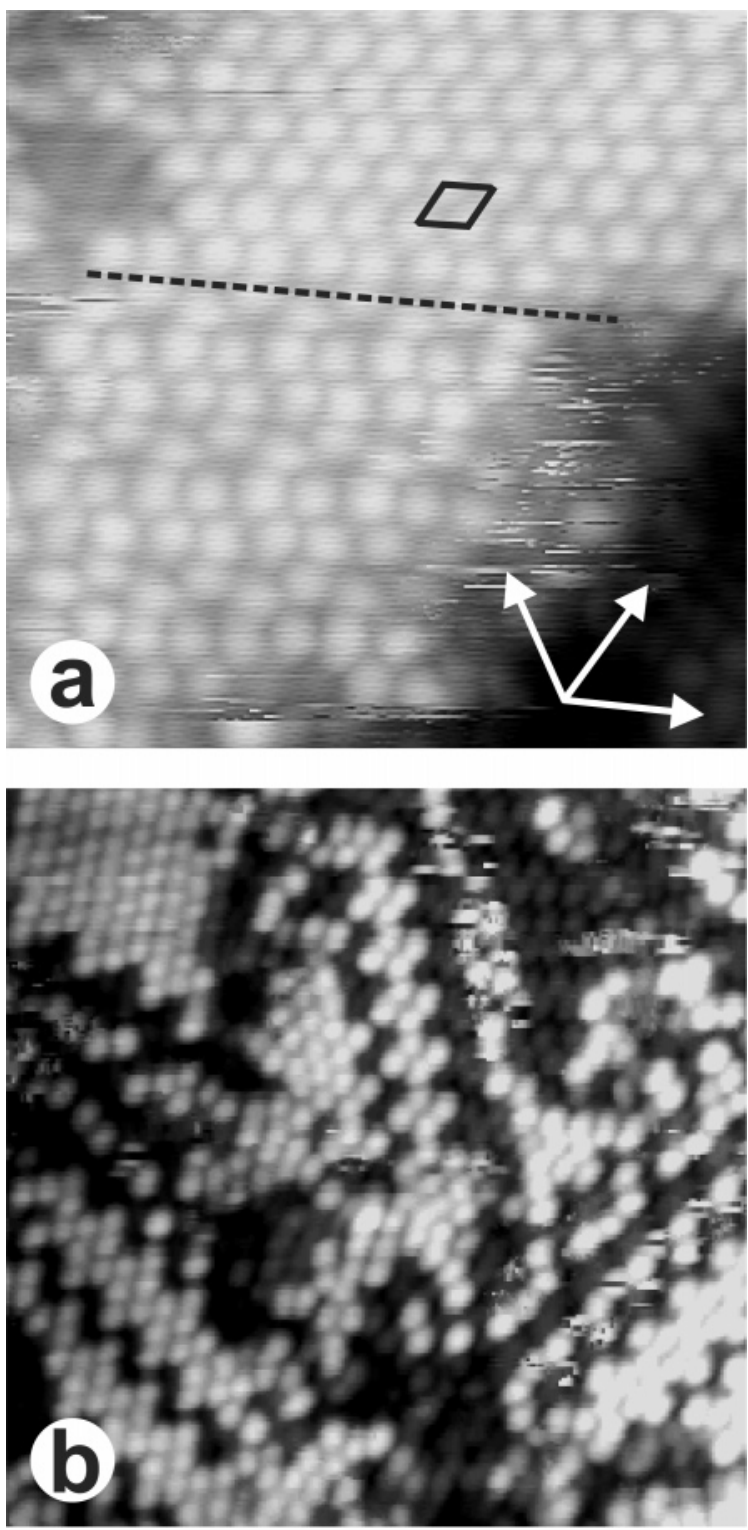

Figure 3. Molecular structures predominant after room-temperature deposition at higher cysteine coverages. (a) Areas of different structures are found, exhibiting several variations of a hexagonal pattern. In the upper part of the image, a more well-defined area is found. Image size: $76 \AA \times 87 \AA$. (b) In some areas, cysteine molecules are apparently missing from the close-packed structure, leading to the appearance of dark rows in the STM image. The faint structures observed between the uppermost protrusions suggest the structures are in fact double or even multiply layered. Image size: $152 \AA \times$ $174 \AA$.

into rows with an additional row of less bright entities situated next them. The distance between the entities of this structure is approximately $5 \AA$ comparable to those found in the previously observed ordered structures. Although this type of pattern is characteristic within the islands, a number of modifications have been observed. In contrast to the situation before the annealing, the molecular island now perturbs the herringbone reconstruction, leading to bending and meandering of the bright reconstruction lines, as observed on the terrace in the vicinity to the island shown in Figure 4.

Annealing to $380 \mathrm{~K}$ of substrates covered at or beyond saturation of the first monolayer leads to results quite different from those observed in the low-coverage regime. After annealing, the majority of the surface is now observed to be covered with a new hexagonal structure, well developed locally but which 


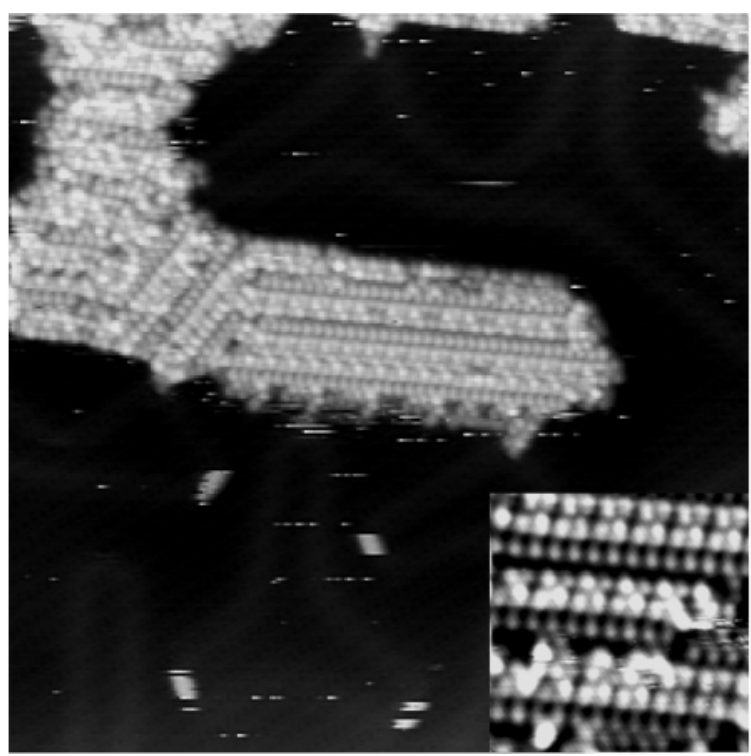

Figure 4. STM images showing internal structure of the cysteine islands after annealing to $380 \mathrm{~K}(381 \AA \times 436 \AA)$. Note the perturbation to the herringbone reconstruction on the surrounding terrace. Inset: Zoom into a characteristic region of the islands (76 $\AA \times 87 \AA$ ).

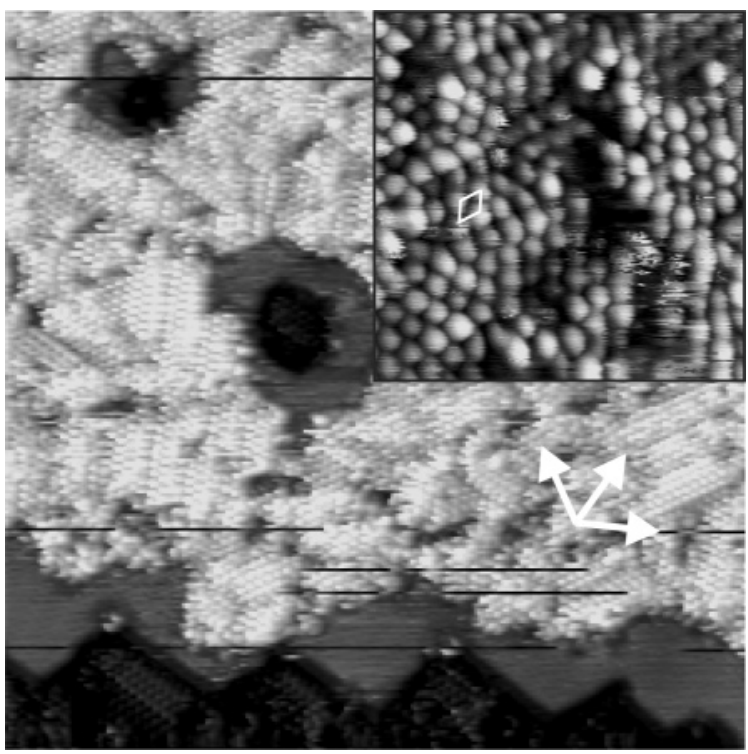

Figure 5. Structure found after annealing in the high coverage regime $(381 \AA \times 436 \AA)$. Locally a $(\sqrt{3} \times \sqrt{ } 3) \mathrm{R} 30^{\circ}$ superstructure is formed as shown in the inset $(76 \AA \times 87 \AA)$. The overlayer is imperfect on a large scale and possesses many monatomically deep holes. Characteristically, the upper edge of the holes and the regions close to the strongly facetted step-edges are free from molecular adsorbates.

tends to form small elongated domains (Figure 5). A highresolution image is displayed in the inset. In contrast to the structures observed at lower temperatures, the high-symmetry directions of this overlayer are always close to $30^{\circ}$ from the close-packed directions of the underlying gold lattice. The distance between the protrusions is $5.6 \pm 0.4 \AA$ which is within $10 \%$ from the distance expected for a $(\sqrt{ } 3 \times \sqrt{ } 3) \mathrm{R} 30^{\circ}$ overlayer. In addition, formation of this structure is accompanied by significant changes of the substrate in terms of pronounced step-faceting and formation of holes with a depth of $2.5 \pm 0.2 \AA$ corresponding well with the step height of $2.35 \AA$ on $\mathrm{Au}(111)$. These observations together strongly indicate that the observed structure is a covalently bound $(\sqrt{ } 3 \times \sqrt{ } 3)$ R30 $0^{\circ}$ molecular overlayer as generally observed for alkanethiol films on $\mathrm{Au}(111){ }^{2}$

\section{Discussion}

In the following we compare the STM results presented above to existing spectroscopic and structural data for cysteine adsorbed on gold surfaces.

A number of XPS measurements of cysteine on gold exist, but they have all been performed under conditions deviating somewhat from the present experiments, requiring some caution in performing direct comparisons. Studies performed on single crystalline $\mathrm{Au}(111)$ films that were exposed to cysteine in aqueous solution and subsequently transferred to the vacuum chamber for XPS measurements ${ }^{9,11}$ have shown split S2p and Au4f peaks, interpreted as arising from covalent $\mathrm{S}-\mathrm{Au}$ binding, along with a nonshifted S2p peak attributed to noncovalently bound sulfur atoms, possibly situated in a second layer with the sulfur atoms oriented away from the surface. Similar conclusions were drawn from experiments where in situ (vacuum) deposition of cysteine was performed on polycrystalline Au substrates. ${ }^{7}$ The existence of unbound sulfur atoms and formation of double layers is consistent with some of the structures observed by STM (Figure $3 b)$. In contrast, the XPS-based observation of covalent $S-A u$ bonding in as-deposited films appears at variance with our conclusion that thermal activation is needed before covalently bound overlayers form. However, the possibility for cysteinegold reaction is likely to be different under aqueous/electrochemical conditions or on more reactive defect-rich polycrystalline substrates. STM-based measurements on liquid-deposited cysteine films have indeed in some instances found $(\sqrt{3} \times \sqrt{ } 3)$ $\mathrm{R} 30^{\circ}$ structures and characteristic etch pits, ${ }^{8,9}$ strongly indicating that formation of structures based on covalent $\mathrm{S}-\mathrm{Au}$ bonds is more facile under conditions deviating from vacuum deposition.

Thermal desorption spectroscopy has been performed for cysteine on $\mathrm{Au}(111)$, showing multilayers of cysteine to desorb at $353-373 \mathrm{~K} .{ }^{17}$ This is consistent with the observation in the present study that only a monolayer remains after annealing to $380 \mathrm{~K}$. In fact, in ref 17 , cysteine monolayers were prepared by multilayer deposition followed by annealing at $373 \mathrm{~K}$ to desorb the cysteine molecules situated in the multilayers. From measurements using spectroscopic techniques based on scattering of low-energy ions, it was concluded from this study that the remaining monolayer consisted of cysteine molecules in zwitterionic form. Since the preparation procedure used in this study corresponds well to our procedure for producing the observed $(\sqrt{3} \times \sqrt{3}) \mathrm{R} 30^{\circ}$ overlayers, it seems likely that this conclusion should also apply to the molecules in that structure. In this respect, it is important to emphasize that the data presented presently shows that the monolayer films produced by the convenient annealing procedure of ref 17 are likely to be quite different in their properties from room temperature as-deposited films (which were not addressed in the study of ref 17).

It is finally relevant to compare the present data to our own extensive dataset for cysteine adsorption on the $\mathrm{Au}(110)-(1 \times$ 2) surface..$^{23-25}$ Also for that system, the observed structures depended critically on the thermal treatment of the sample, and it was concluded that thermal activation to $370 \mathrm{~K}$ was necessary before covalent $\mathrm{S}-\mathrm{Au}$ interaction occurred (see however also

(23) Kühnle, A.; Linderoth, T.; Hammer, B.; Besenbacher, F. Nature 2002 415,891

(24) Kühnle, A.; Linderoth, T.; Besenbacher, F. J. Am. Chem. Soc. 2003, 125 14680 .

(25) Kühnle, A.; Molina, L. M.; Linderoth, T. R.; Hammer, B.; Besenbacher, F. Phys. Rev. Lett. 2004, 93, 086101. 
ref 26). Interestingly, although in the studies of cysteine/Au(110) effects related to the chiral nature of the cysteine molecules were abundant, no sign of molecular chirality have been found in the present data. We ascribe this to the lack of submolecular resolution in the present study in combination with the fact that all of the observed structures have a high degree of symmetry.

Perhaps the most striking feature of the present results is the multitude of coexisting structures observed upon room temperature deposition, in particular amorphous islands versus highly crystalline, extended domains. We speculate that these qualitatively different structures may reflect different charge states of the adsorbed cysteine molecules, i.e., zwitterionic versus noncharged, in combination with the fact that there is no obviously preferred molecular adsorption geometry for the cysteine molecules, in contrast, e.g., to molecules of planar geometry. There are a multitude of possibilities for specific intermolecular interactions between adsorbed cysteines, in particular hydrogen bonding between $-\mathrm{COOH}$ groups of noncharged adspecies and between $-\mathrm{NH}_{3}{ }^{+}$and $-\mathrm{COO}^{-}$groups of molecules in the zwitterionic state. In addition, the $-\mathrm{SH}$ group may also participate in hydrogen bond formation for molecules that are not deprotonated and covalently bound to gold. As a result, pronounced polymorphism ensues. Since our data does not provide any clues to differentiate between these different scenarios, we do not find it justified to formulate detailed models for the observed structures. We note, however, that the observed crystalline overlayer of quadratic symmetry (Figure 2) bears some resemblance to a structure found for bulk cysteine using X-ray diffraction. Here the monoclinic unit cell has a lateral extent of $9.44 \AA \times 11.34$ $\AA\left(\beta=109^{\circ}\right)$ and contains four cysteines in zwitterionic form bound by $\mathrm{N}-\mathrm{H}-\mathrm{O}$ hydrogen bonds. ${ }^{27}$

\section{Summary and Conclusion}

In summary, we have used in situ STM to investigate adsorption structures found upon vapor deposition of the amino acid cysteine

(26) Gonella, G.; et al. J. Phys. Chem. B 2005, 109, 18003.

(27) Görbitz, C. H.; Dalhus, B. Acta Crystallogr. C 1996, 52, 1756. onto a single-crystal Au(111) surface under UHV conditions, comparing the as-deposited room temperature structures to those obtained after thermal annealing of the substrate to approximately $380 \mathrm{~K}$. A variety of different structures were observed depending upon both temperature and coverage. At room temperature and at submonolayer coverages, islands with no apparent molecular order were observed to coexist with a highly ordered molecular layer of quadratic symmetry. Upon increasing coverage, a multitude of slightly differing structures with short-range hexagonal order were observed. In neither the quadratic nor the hexagonal overlayer structures, any epitaxial or rotational relationship to the underlying $\mathrm{Au}(111)$ substrate was observed, suggesting that no specific molecular adsorption positions on the gold surface are involved in the formation of these structures. Upon annealing to $380 \mathrm{~K}$, pronounced changes in the adsorption structures and surface morphologies were observed. Most importantly, the well-known $(\sqrt{3} \times \sqrt{ } 3) \mathrm{R} 30^{\circ}$ superstructure characteristic for alkanethiol self-assembled monolayers on Au(111) developed upon annealing of substrates with cysteine coverages beyond saturation of the first monolayer. Formation of this overlayer was accompanied by development of monatomically deep etch pits which is also typically observed upon formation of alkanethiol self-assembled monolayers.

No definite conclusion pertaining to the binding properties of cysteine to the $\mathrm{Au}(111)$ surface can be drawn from these structural STM data alone. The results do suggest, however, that covalent $\mathrm{S}-$ Au bonding only occurs upon thermal activation of the vacuum deposited cysteine molecules. The observation of several coexisting structures in the room-temperature as-deposited films is furthermore important input to future experiments using nonlocal, spectroscopic techniques since in such cases an average over these qualitatively different structures will necessarily always be performed.

Acknowledgment. This work was supported by the Danish National Research Foundation, the Danish Natural Science Research Council and the Carlsberg Foundation. 